\title{
Adam J. DAVIS, The Medieval Economy of Salvation: Charity, Commerce, and the Rise of the Hospital
}

\section{Arnaud Baudin}

\section{OpenEdition}

\section{Journals}

Édition électronique

URL : https://journals.openedition.org/ccm/8765

DOI : $10.4000 / \mathrm{ccm} .8765$

ISSN : 2119-1026

\section{Éditeur}

Centre d'études supérieures de civilisation médiévale/Université de Poitiers

\section{Édition imprimée}

Date de publication : 1 décembre 2021

Pagination : 388-391

ISBN : 978-2-490783-11-3

ISSN : 0007-9731

Référence électronique

Arnaud Baudin, «Adam J. DAvIs, The Medieval Economy of Salvation: Charity, Commerce, and the Rise of the Hospital », Cahiers de civilisation médiévale [En ligne], 256 | 2021, mis en ligne le 01 décembre 2021, consulté le 03 juin 2022. URL : http://journals.openedition.org/ccm/8765; DOI : https://doi.org/ $10.4000 / \mathrm{ccm} .8765$

\section{cc) (i) (9)}

La revue Cahiers de civilisation médiévale est mise à disposition selon les termes de la Licence Creative Commons Attribution - Pas d'Utilisation Commerciale - Pas de Modification 4.0 International. 
Adam J. Davis, The Medieval Economy of Salvation: Charity, Commerce, and the Rise of the Hospital, Ithaca/Londres, Cornell University Press, 2019.

Professeur à l'Université de Denison (Ohio), Adam J. Davis s'intéresse à la vie religieuse, aux attitudes médiévales à l'égard du commerce, ainsi qu'à l'histoire des œuvres de bienfaisance et des institutions caritatives. Dans la continuité de ses travaux récents, son dernier ouvrage poursuit l'exploration de l'interaction entre les idées et les institutions médiévales, les valeurs et les pratiques sociales et propose d'évaluer le lien entre l'émergence de l'économie du commerce médiéval et la montée de nouvelles formes caritatives dans l'Occident latin (introduction, p. 1-32). À partir du laboratoire offert par le comté de Champagne aux $\mathrm{XII}^{\mathrm{e}}$ et $\mathrm{XIII}^{\mathrm{e}} \mathrm{s}$., l'auteur interroge la manière dont l'activité économique engendrée par le cycle des quatre foires annuelles de Troyes, Provins, Lagny et Bar-sur-Aube a favorisé en parallèle l'essor des hôpitaux dans ces villes et, au-delà, dans le réseau urbain (Reims, Châlons, Meaux, etc.) et rural (léproseries) environnant. Les sources écrites, nombreuses et souvent inédites de cet âge d'or champenois (cartulaires, chartes de donation, rapports de visites, livres de comptes, etc.), renseignent sur les protecteurs de ces institutions, leurs sources de revenus, leur organisation, leur personnel et les malades accueillis. Dans la lignée des travaux de Nicole Bériou notamment, l'auteur s'attache à montrer que la charité revient de manière récurrente dans le corpus des prédicateurs des $\mathrm{XII}^{\mathrm{e}}$ et $\mathrm{XIII}^{\mathrm{e}}$ s. (chap. 1 : "Medieval $^{2}$ Understandings of Charity: From Penance to Commerce », p. 33-78). À partir de l'Évangile de Matthieu (25:35-40), un certain nombre d'universitaires et de théologiens, au premier rang desquels Pierre le Chantre et Jacques de Vitry, définissent le don comme joyeux, désintéressé, silencieux et discret. Les sermons des théologiens sont nourris d'exempla définissant les modèles à éviter ou à imiter. Les développements autour de la nature du don, dans lesquels la critique des indulgences n'est jamais loin (Pierre Abélard), s'accompagnent d'une réflexion sur la valeur de celui-ci. Dans le contexte de l'essor commercial, certains canonistes filent rapidement la métaphore, plaçant la charité au cœur d'une économie du Salut qui, tout en critiquant l'usure, considère le don aux pauvres comme participant d'une forme d'échanges. Cette théologie de la charité se retrouve chez Innocent III (Libellus de eleemosyna) pour qui le pauvre accorde au riche des profits supérieurs puisqu'en échange du don matériel ce dernier reçoit la récompense éternelle. Thomas de Chobham distingue le mauvais du bon usurier qui, en accomplissant la charité, reçoit ses intérêts dans l'Au-delà. Laurent d'Orléans (La somme 
le roi) définit les dons aux pauvres comme des prêts à Dieu. Mais de nombreux autres théologiens estiment l'Église en danger lorsqu'elle place l'économie du Salut au cœur de son discours sur la charité. L'autre question fondamentale est celle de la science de la caritas chère à Thomas d'Aquin. En réalité, cette voie de la charité désintéressée est donnée par ces laïcs qui, dans les pas du Poverello, se dévouent au service des malades (Marie d'Oignies, Élisabeth de Hongrie, Jean de Montmirail, Marguerite de Bourgogne, Louis IX) et dans lesquels, comme le prêtre à l'autel, ils voient la présence réelle du Christ.

La Champagne offre donc un modèle de ces paysages de la charité qui se mettent en place à partir du $\mathrm{XII}^{\mathrm{e}}$ s. en Occident (chap. 2 : «The Creation of a Charitable Landscape », p. 79-114), alors même que l'extraordinaire réussite du commerce forain dans les domaines Thibaudiens a aussi pour effet collatéral l'augmentation de la pauvreté. En 1299, le doyen du chapitre cathédral de Troyes lègue 20 livres tournois à distribuer aux pauvres le jour de ses funérailles, soit un denier chacun, somme qui aurait pu bénéficier à 4800 personnes sur une population urbaine estimée alors à 20000 .

La dynastie comtale, suivie par le réseau vassalique, est à l'initiative de cette politique de la charité comme en attestent la plupart des testaments princiers attribuant des sommes à distribuer aux pauvres (Henri I ${ }^{\text {er }}$, Thibaud V) ou fondant des hôpitaux (Château-Thierry par Jeanne de Navarre en 1305). Le Libéral transforme la cité épiscopale de Troyes en capitale politique et économique tout en favorisant les chapitres canoniaux, les hôpitaux et les léproseries sur son domaine. Fondé v. 1157, l'hôtel-Dieu de Troyes est le principal établissement hospitalier de la ville. Longtemps lié financièrement et administrativement au chapitre Saint-Étienne, l'hôpital, autonome en 1263, prend le nom d'Hôtel-Dieu-le-Comte.

Outre la léproserie des Deux-Eaux, fondée à 1123 au sud de la ville, Troyes dispose encore de quatre autres établissements hospitaliers (Saint-Nicolas, SaintAbraham, Saint-Bernard et Saint-Esprit). À Provins, l'hôtel-Dieu, fondé vers le milieu du XI s. s., jouit d'une grande réputation, Jacques de Vitry le plaçant au même rang que ceux de Paris ou de Bruxelles. Comme à Troyes, l'hôpital du Saint-Esprit est attaché aux chanoines de Montjoux pour l'accueil des pèlerins. À Bar-sur-Aube, l'hôpital Saint-Nicolas, un établissement mixte, existe dans les années 1130.

La Champagne joue un rôle essentiel dans la diffusion du monachisme réformateur qui assume largement sa part de la charité en milieu rural (porterie de Clairvaux). À la suite des travaux d'Anne Lester
(Creating Cistercian Nuns: The Women's Religious Movement and Its Reform in Thirteenth-Century Champagne, Ithaca/New York, Cornell University Press, 2011), A. Davis rappelle que les couvents de moniales cisterciennes en Champagne ont souvent pour origine un hôpital ou une léproserie (Val-desVignes). Loin de faire concurrence, le réseau monastique vient en soutien des petits établissements dont les moyens financiers ne sont plus suffisants. L'hôpital de Brienne, fondé v. 1138, est rattaché aux Augustiniens de Saint-Loup (1206), puis aux Bénédictins de Montier-en-Der (1224), enfin aux Teutoniques de Beauvoir (1230).

Les bienfaiteurs des hôpitaux champenois, membres de la dynastie comtale, aristocrates ou bourgeois, attendent en retour l'assurance des fruits portés par leurs dons (chap. 3 : " Hospital Patrons and Social Networks », p. 115-163).

Les hôpitaux, confrontés à l'émergence des ordres mendiants, demeurent ceux qui, au XIII ${ }^{\mathrm{e}}$ s., profitent le plus des effets de la charité : $44 \%$ des testaments flamands contiennent un legs en faveur d'un hôpital. Si certains établissements champenois connaissent une diminution des dons, certains comme celui de Provins atteignent des sommets dans les années 1260 à 1280 . La révolution charitable concerne tout le monde, une évolution du milieu social des donateurs qui est particulièrement évidente à Saint-Nicolas de Troyes, soutenue majoritairement par la bourgeoisie troyenne.

Des liens sociaux, familiaux et géographiques transparaissent dans le choix du bénéficiaire : l'hôpital soutenu par plusieurs générations d'un même lignage, celui auquel appartient un membre de la famille, celui où l'on a été soigné ou encore où l'on souhaite être inhumé. Mais de nombreux testaments sont totalement désintéressés, favorisant plusieurs institutions en même temps, sans lien d'aucune sorte.

Si la formule «pro anima » tend à disparaître au XIII" $\mathrm{s}$. des dons aux abbayes, elle reste très présente dans les testaments en faveur des hôpitaux ( 86 \% dans ceux de l'hôpital de Provins), en lien avec l'augmentation des legs réalisés en contrepartie de messes anniversaires. À l'Hôtel-Dieu-le-Comte, seuls $18 \%$ des donateurs promettent des anniversaires à la fin $\mathrm{du} \mathrm{XII}^{\mathrm{e}} \mathrm{s}$., chiffre qui atteint $44 \%$ après 1250 . Les chartes mettent aussi en évidence le souhait d'être enterré dans le cimetière de l'hôpital, source de conflits avec la paroisse voisine. En 1195, Célestin III autorise l'hôpital de Provins à posséder son cimetière à condition que l'établissement obtienne l'accord de l'archevêque de Sens et des curés de la ville. 
Enfin, si ces dons sont accomplis en faveur des pauvres et des malades, certaines chartes mettent en avant les besoins des hôpitaux et la qualité du travail qui y est accompli. Des rentes en argent sont instituées pour nourrir et vêtir les pauvres ou dédiées à l'achat de linceuls. En juin 1270, le chevalier Renaud de Bar donne sa maison située juste à côté l'hôtel-Dieu de Troyes pour qu'y soit fondé un petit hôpital pour l'accueil de six femmes enceintes.

Les livres de comptes témoignent de la bonne gestion des hôpitaux (chap. 4 : «Managing a Hospital's Property », p. 164-186). Si quelques-uns ont moins de 20 livres de revenu annuel (Coulommiers, Payns), d'autres comme les hôtels-Dieu de Troyes et de Provins génèrent entre 1000 et 2000 livres par an. En 1300, Saint-Nicolas produit des quantités importantes de céréales, stockées dans des granges, possède un troupeau de 300 têtes, un cellier, un lardier, des écuries. L'hôpital, également actif sur le marché des rentes, collecte alors 38 livres sur 15 maisons et chambres qu'il possède en ville. Des rentes parfois réinvesties lors des foires, mais aussi dans l'achat de denrées, la réparation et l'entretien des bâtiments et des maisons. Les principaux hôpitaux urbains sont de puissants seigneurs fonciers, disposant de fiefs reçus en dons, aliénés du domaine comtal et amortis grâce à la libéralité du prince. Ils reçoivent ou achètent des dîmes et peuvent pratiquer le crédit. Parfois, ces prêts sont eux-mêmes transformés en dons, notamment lorsqu'ils sont consentis à une autre institution charitable : en 1244, l'hôpital Saint-Abraham de Troyes prête 50 livres à la léproserie des Deux-Eaux, puis annule cette dette.

L'origine sociale du personnel hospitalier est diverse (chap. 5 : " "In Service of the Poor": Hospital Personnel in Pursuit of Security ", p. 188-240). La volonté de servir les pauvres et les malades est la motivation principale même si certains théologiens dénoncent les intentions cyniques de ceux cherchant à se mettre à l'abri du besoin en prenant prétexte de la charité. L'obituaire de Provins mentionne v. 1280 ce maître Jean Furnerius, la plus ancienne mention d'un physicien en France avant le XIV ${ }^{\mathrm{e}} \mathrm{s}$. Le chapelain, à la fois prêtre et frère servant, célèbre la messe, les heures liturgiques, donne les sacrements, prêche et participe aux soins. Le maître (magister), chef de la communauté spirituelle choisi au sein de celle-ci, a des fonctions de directeur. Viennent ensuite les simples frères et sœurs, qui prononcent les vœux à leur entrée, et dont les statuts de chaque établissement prévoient précisément le nombre; les conversi qui rejoignent l'hôpital à la fin de leur vie pour s'occuper des malades; certains couples mariés, même si canons conciliaires et statuts l'interdisent au XIII ${ }^{\mathrm{e}} \mathrm{s}$. ; les donati - souvent des veuves - qui se donnent à l'hôpital avec leurs biens; des serfs, enfin, comme ces 40 donnés en 1189 par le comte Henri II à l'hôtel-Dieu de Troyes.

Les sources des $\mathrm{XII}^{\mathrm{e}}$ et $\mathrm{XIII}^{\mathrm{e}} \mathrm{s}$. parlent davantage des dons que de la nature des soins apportés aux malades (chap. 6: «The Sick Poor and the Economy of Care », p. 241-275). Il faut alors se tourner vers l'archéologie. La fouille de la place de la Libération à Troyes, entre 2004 et 2008, révéla 2500 tombes, dont une partie relevant du cimetière de l'hôtel-Dieu, délivrant des informations de premier ordre sur les patients et leurs pathologies. Outre les vagues épidémiques, la majorité des cas montrent des infections - la tuberculose en particulier -, et des fractures osseuses.

À l'hôtel-Dieu de Troyes, les malades sont accueillis dans une infirmerie, séparée des espaces pour les pauvres. Selon les établissements, on reçoit aussi des femmes enceintes, des enfants abandonnés, des pèlerins, des voyageurs, d'anciennes prostituées, mais aussi des clercs âgés et des étudiants pauvres. SaintNicolas de Troyes accueille des étudiants, à l'image de la maison des Bons-Enfants à Reims, qui se mettent en échange au service de l'hôpital. Vécue comme une punition divine, la maladie est d'abord soignée par les sacrements, l'assistance à l'Eucharistie et aux différents offices depuis son lit, la confession et la communion, à quoi s'ajoute la vénération des nombreuses reliques possédées par les hôpitaux ou le décor peint qui offre des images de dévotion et de méditation aux malades comme à Tonnerre, Angers ou Laon.

L'assistance s'exerce aussi à l'extérieur des hôpitaux où vivent $10 \%$ de sans-abris. Deux fois par semaine, l'hôpital d'Angers envoie des frères à leur rencontre, en complément des confréries et guildes paroissiales qui se développent dans l'ensemble de l'Occident au XIII ${ }^{\mathrm{e}} \mathrm{s}$. À la foire de Saint-Rémi de Troyes, 1'hôtel-Dieu distribue 20 sous de pitance au-delà des remparts, ainsi qu'aux pauvres du bourg de Saint-Augustin au sud de la ville.

Au final, A. Davis livre, dans un style élégant et clair, un ouvrage d'une grande érudition qui apporte un éclairage nouveau sur la nature de la charité, la manière dont elle est perçue, vécue et exercée par l'ensemble de la population au cours de la première grande période d'essor économique de l'Occident. Il montre de manière convaincante comment les hôpitaux, bénéficiant de conditions favorables, mettent en place une économie de la charité qui n'occulte pas la critique de l'usure par les moralistes et la montée des inégalités. V. 1300, alors que les foires commencent leur long déclin, les hôpitaux 
créés dans la Champagne du XII ${ }^{\mathrm{e}} \mathrm{s}$. sont devenus des institutions solides, ancrées dans le paysage urbain, prêtes à affronter la longue période de guerre, de famines et d'épidémies qui s'ouvre, apportant avec elle son lot de vagabonds et de miséreux.

Arnaud BAUDIN

LAMOP - UMR 8589/

Archives du Patrimoine de l'Aube 